\title{
MS01-05 | Measuring The Dose: Photoelectron Escape In Micro-Crystals
}

Storm, Selina (Diamond Light Source, Didcot, GBR); Crawshaw, Adam (Diamond Light Source, Didcot, GBR); Devenish, Nicholas (Diamond Light Source, Didcot, GBR); Bolton, Rachel (University of Southampton, Southampton, GBR); Tews, Ivo (University of Southampton, Southampton, GBR); Evans, Gwyndaf (Diamond Light Source, Didcot, GBR)

With the trend of using microcrystals and intense microbeams, radiation damage becomes a more pressing problem. Theoretical calculations by Nave and Hill [1] show that the photoelectrons that primarily cause damage can escape very small crystals, reducing the effective dose, an effect which was demonstrated to be pronounced at higher energies [2].

To investigate photoelectronic escape, we measured radiation damage at cryo-temperatures on lysozyme crystals of 5um and 20um mounted on a cryo-EM grid. The data were collected at $13.5 \mathrm{keV}$ and $20.1 \mathrm{keV}$ using a $2 \mathrm{M} \mathrm{CdTe}$ Pilatus and were analysed with DIALS [3] and RADDOSE3D [4]. Our data indicate a longer crystal lifetime for smaller crystals and support the theory of photoelectron escape.

[1] Nave \& Hill, (2005), J. Synchrotron Rad. 12, 299-303

[2] Sanishvili et al. (2011), PNAS 108(15), 6127-6132

[3] Winter et al. (2018), Acta Cryst. D74, 85-97

[4] Zeldin et al. (2013), J. Appl. Cryst. 46, 1225-1230 